\title{
De la répulsion à la fascination : l'Internet et les représentations des NTIC
}

\section{Viviane Serfaty}

\section{(2) OpenEdition \\ Journals}

Édition électronique

URL : http://journals.openedition.org/asp/2128

DOI : 10.4000/asp. 2128

ISBN : 978-2-8218-0382-4

ISSN : 2108-6354

Éditeur

Groupe d'étude et de recherche en anglais de spécialité

Édition imprimée

Date de publication : 31 décembre 2000

Pagination : 231-241

ISSN : 1246-8185

\section{Référence électronique}

Viviane Serfaty, «De la répulsion à la fascination : I'Internet et les représentations des NTIC », ASp [En ligne], 27-30 | 2000, mis en ligne le 28 janvier 2011, consulté le 02 mai 2019. URL : http:// journals.openedition.org/asp/2128; DOI : 10.4000/asp.2128

Ce document a été généré automatiquement le 2 mai 2019.

Tous droits réservés 


\title{
De la répulsion à la fascination : l'Internet et les représentations des NTIC
}

\author{
Viviane Serfaty
}

1 L'Internet ${ }^{1}$ se construit, depuis plusieurs années, en champ à part entière, selon les analyses de Pierre Bourdieu dans le domaine de l'art et de la littérature. Celui-ci souligne en effet que l'émergence d'un champ est perceptible lorsqu'on peut en définir les enjeux spécifiques et les luttes qui leur sont liées, d'une part et d'autre part lorsqu'apparait « un corps de conservateurs » dédié à répertorier et archiver tout ce qui se rapporte au champ nouvellement constitué2.

Ces caractéristiques s'observent pour l'Internet, qui non seulement suscite des interprétations contradictoires de la part des acteurs qui y sont impliqués, mais est aussi l'objet d'une immense quantité de discours aussi bien parmi les chercheurs universitaires que dans l'ensemble de la société. La constitution de l'Internet en champ à part entière se perçoit aussi dans les discours produits à son sujet par les médias plus anciens. Alors même que les groupes de presse et les chaînes de télévision se bousculent pour faire connaître leur présence sur le réseau, les journalistes font de l'Internet un vecteur de rumeurs et d'informations non fondées. L'unanimité dans le dénigrement du réseau par les journalistes de la presse écrite ou de la télévision révèle que l'Internet semble soudain être devenu un rival suffisamment menaçant pour qu'on élabore des stratégies capables d'en amoindrir l'influence, processus qui n'est pas sans rappeler l'action entreprise par les journaux lors de l'émergence de la radio en tant que média de masse pendant les années vingt et trente. La constitution de l'Internet en tant que champ rend essentielle l'analyse des discours qui accompagnent cette nouvelle technologie, afin d'en définir les caractéristiques saillantes et d'en déterminer les fonctions. 


\section{Corpus}

3 Pour identifier les structures profondes de la vision contemporaine de l'Internet, nous avons choisi d'étudier d'une part les chroniques écrites par les créateurs du réseau ainsi que par ses premiers utilisateurs aux États-Unis. D'autre part, nous avons analysé un corpus de textes constitué de 630 brefs essais rédigés, lors de l'épreuve d'anglais, par les candidats au concours d'entrée en première année à l'Institut d'études politiques de Strasbourg en 1997. Les rédacteurs du sujet avaient choisi de soumettre à la sagacité des candidats la question suivante: "Quelles sont vos craintes et vos attentes par rapport à l'Internet? ». Nous avons pu constater que la perception du réseau s'organise le long de lignes de force très semblables parmi les jeunes gens dont nous avons étudié les énoncés comme parmi les auteurs qui décrivent et analysent leur vécu de la communication médiatisée par ordinateur. Cette convergence nous a permis de vérifier notre hypothèse de départ, selon laquelle la perception du réseau n'avait rien d'une expérience de première main, mais se constitue comme représentation sociale de la communication, des technologies qui lui sont associées et du rôle qu'elles jouent dans l'autodéfinition d'une société donnée.

4 Nous émettons l'hypothèse que ces deux types de texte nous offriront un instantané de l'imaginaire dans lequel l'Internet vient se loger. Notre propos n'est pas de connaître les sujets qui s'expriment ou de percevoir leur singularité, mais plutôt de dégager leurs représentations, les diverses stratégies dont ils usent afin de donner du sens aux transformations sociales dont ils sont les contemporains et quelquefois les acteurs. L'expression singulière est employée comme moyen de repérage d'un contenu latent qui est, lui, sinon socialement déterminé, du moins reflet de représentations sociales. Ces représentations produisent des effets de réel considérables dans la mesure où elles tendent à modeler les comportements et les usages face à l'Internet. Leur analyse s'avère donc d'importance cruciale.

5 L'analyse de contenu de notre corpus a mis en relief l'ambivalence du discours sur l'Internet, une ambivalence telle qu'elle s'apparente à des conflits d'imaginaires. Le clivage qui marque ces discours n'est pas seulement dû à l'intitulé de la question, mais à la relation à l'objet technologique lui-même. Il témoigne de l'existence d'une lutte qui est avant tout lutte symbolique, et dont l'enjeu est le sens à attribuer à l'Internet.

\section{Utopie}

6 Le clivage qui traverse le discours sur l'Internet doit être relié à l'utopie ainsi qu'à l'antiutopie dans leurs multiples dimensions. Le réseau est perçu et décrypté à l'aide de ces structures antinomiques, dont la longue histoire informe et modèle la perception de cet objet éminemment contemporain.

7 Les représentations utopiques concourent, grâce à l'élaboration d'un mythe desorigines, à faire de l'Internet le lieu d'un renouveau au travers de la notion de retournement ${ }^{3}$. Celleci s'applique à la propriété, décriée au profit de la gratuité, ainsi qu'au pouvoir, mis en échec par la notion d'égalité pour tous. Le retournement de l'identité individuelle joue lui aussi un rôle non négligeable. Dans la version utopique de cette notion, le corps que chacun a reçu en partage perd son rôle crucial dans la définition de l'identité sociale ou 
psychique : chacun peut se recréer selon son désir par la grâce de quelques lignes de texte. Mais cette projection, si elle est source de fascination, contient aussi un risque, celui d'ébranler l'unité du moi, si bien que dans ce cas précis, fascination et répulsion se rejoignent.

Les représentations anti-utopiques s'attachent principalement aux notions d'espace et de corps : les représentations anti-utopiques de l'espace conduisent d'une part à redouter la perte de l'intimité, l'affaiblissement des barrières de classe ainsi que la prolifération de la présence d'autrui. D'autre part, la notion même d'espace virtuel suscite la crainte d'une prolifération des espaces qui favorise l'incertitude et fait de l'Internet le concurrent du monde réel.

Les représentations anti-utopiques du corps tel que l'Internet le médiatise font principalement intervenir la notion de dématérialisation du corps: on craint sa transformation en pur signifiant, ce qui introduit une dimension d'indétermination propice à toutes sortes d'anxiétés de dissolution et de perte : c'est le pôle de la répulsion.

L'analyse de contenu fait émerger trois grands paradigmes, tous traversés par le clivage utopie/anti-utopie: le paradigme culturel et communicationnel, le paradigme de l'anarchie et le paradigme spatio-temporel.

\section{Le paradigme culturel et communicationnel}

11 Le paradigme culturel est indissolublement lié au paradigme communicationnel, puisque l'Internet est perçu comme moyen universel d'accéder à une culture elle aussi universelle, qui ne tient aucun compte du temps du processus d'apprentissage ou de la dimension sociale de l'acquisition et de la transmission des savoirs. Les notions de culture et de communication apparaissent avec une régularité extrême, comme marqueurs de distinction symbolique, pour $90 \%$ de l'échantillon.

L'affirmation de la possibilité d'un accès universel à la culture et au savoir constitue l'un des lieux nodaux de jonction avec l'utopie : le désir de savoir universel, de savoir total, sans entraves s'apparente au désir de transparence qui est l'une des caractéristiques de l'univers utopique, où chaque élément de la cité doit traduire et exprimer le plan d'ensemble. Sur le plan de l'imaginaire personnel, transparence et savoir correspondent à un désir d'union parfaite, de communication sans faille, proche de nos toutes premières expériences de fusion avec le corps maternel.

Deuxième point de jonction majeur avec l'utopie : le gouvernement de savants qui, chez Saint-Simon ou Francis Bacon, exercent le pouvoir pour le bien de tous. Dans le cas de l'Internet tel qu'il est rêvé, il s'agit de remplacer le pouvoir politique par le pouvoir de la connaissance et notamment de la connaissance de l'informatique. Le caractère universel du pouvoir de la connaissance est réaffirmé, de même que sa souveraineté sur le pouvoir politique à l'origine, lui, des dissensions et de l'hostilité entre les peuples et d'emblée suspecté de tendre vers la tyrannie. En effet, le savoir est paré d'une auréole de pureté qui le distingue radicalement de tous les autres domaines de l'expérience: il est conçu comme indépendant des passions humaines, à même de dissiper les brumes d'ignorance qui nous empêchent de percevoir le monde. Dans cette optique également, le savoir est perçu comme une entité en soi, préexistante et neutre, attendant d'être dévoilée au fur et à mesure du développement de l'entendement humain. Enfin, le savoir n'est pas perçu 
comme source de pouvoir sur autrui, à l'instar du politique, mais comme élargissement de la stature de chaque être humain.

Il importe de souligner qu'il n'existe pas de versant anti-utopique dans ce qui touche l'accès à la culture ; pour la communication, comme nous l'avons déjà évoqué plus haut, l'anti-utopie met en avant la prolifération de la présence d'autrui, la perte d'intimité et l'universelle surveillance.

\section{Le paradigme de l'anarchie (liberté, égalité, sociabilité)}

\section{Principe de liberté}

15 L'Internet, nous l'avons vu, est « le lieu de projection de toutes les ambivalences sociales » (Jeanneret 1998 : 37), si bien que la survalorisation de la communication et de la culture s'accompagne de la vision du réseau en tant que "nouvelle frontière ", dépourvue de lois ou de systèmes capables de la faire respecter. Ce n'est cependant là qu'un aspect du paradigme de l'anarchie, celui qui, en donnant expression à la peur de l'indéterminé, justifie toutes les demandes de contrôle et de répression.

16 L'autre versant du paradigme, utopique celui-là, est celui de l'espace de liberté égalitaire qui offre à la démocratie un champ d'action illimité. Pour de très nombreux commentateurs, «ce que les gens créent sur l'Internet, c'est une démocratie [...] nonreprésentative qui transcende l'État-nation» (Nguyen \& Alexander 1996: 111, notre traduction). Certains décrivent ainsi des logiciels qui permettent de faire participer les membres de certains forums à la définition des politiques publiques des gouvernements, ce qui serait une « anticipation [...] de l'idéal d'une démocratie authentique » (Mathias 1997 : 47) : une technologie censée éviter, donc, toute dérive plébiscitaire ou populiste, puisque située en amont du processus de prise de décision politique. De façon semblable, on critique la démocratie représentative qui aboutirait à la dé-responsabilisation du citoyen pour promouvoir une démocratie directe fondée sur l'accessibilité de l'information grâce à l'Internet (Grossman 1995), puisque dans l'idéologie de la communication, l'accès à une information de qualité engendrerait un plus fort engagement en politique (Bimber 1998). Le règne actuel du sondage d'opinion serait poussé à sa logique extrême et permettrait à chacun de faire partie d'un vaste processus consultatif qui l'intégrerait au processus de prise de décision politique en évitant la médiation des partis politiques. Le pôle de la répulsion s'appuie sur le danger de populisme et va jusqu'au pôle de la fascination vis-àvis de l'idéal d'un citoyen omnicompétent, capable de réactions raisonnées quel que soit les problèmes soulevés par la vie publique.

\section{Principe d'égalité}

17 Le paradigme anarchique pousse jusqu'au bout la métaphore contenue dans le mot réseau et par là même la vision déterministe de la technologie, puisque le mode de communication en réseau est censé saper les hiérarchies traditionnelles, verticales, et imposer un mode de communication égalitaire, horizontal. Même la transmission des données par paquets est mise à contribution en tant que métaphore du nivellement de toutes les informations, mais aussi en tant que vecteur de résistance au contrôle, avatar libertaire de ce qui était à l'origine un objectif militaire avoué. On considère que l'information ainsi fragmentée devient impossible à étouffer (Shade 1996:24). Le 
paradigme de l'anarchie s'ordonne autour de la capacité émancipatrice du réseau en raison de la liberté d'expression qu'il permet sur un plan individuel, mais aussi politique et social.

L'exemple de la RFC 1591, dans laquelle est exposée la procédure de mise en place des noms de domaine, fournit une illustration majeure de la volonté d'égalité qui a informé l'attitude des fondateurs du réseau: Jon Postel qui fut, rappelons-le, l'un de ces jeunes chercheurs dont les travaux contribuèrent notablement à la création du réseau, prend soin d'énoncer les principes suivants :

Les responsables d'un domaine [...] ont le devoir de servir la communauté. [...] Ils n'ont pas à se préoccuper des «droits » à un domaine ou des «titres de propriété ». Ils doivent se préoccuper de leur responsabilité vis-à-vis de la communauté et la servir. Le responsable doit être équitable envers tous les groupes qui sollicitent des noms de domaine. Les mêmes règles seront appliquées à toutes les requêtes, toutes les requêtes doivent être traitées également, et les utilisateurs universitaires, commerciaux ou autres seront traités de la même façon ${ }^{4}$.

19 Deux axes majeurs sont présents dans ce bref extrait: le principe d'égalité, qui ne souffre aucune exception, et le principe de service à la collectivité, qui doit supplanter tout désir de pouvoir personnel, ainsi que toute velléité d'appropriation de ces espaces virtuels. C'est l'utopie de type libertaire et anarchiste qui a présidé aux commencements du réseau, et que beaucoup d'utilisateurs de la première heure voient disparaître à regret.

Le paradigme de l'anarchie permet de voir dans le média lui-même l'instrument d'une prise d'autonomie des citoyens/consommateurs vis-à-vis du complexe industriel. Ainsi, Ronda Hauben (1997) retrace la campagne de courrier électronique qui, en 1981, a permis de révéler l'existence d'un défaut de fabrication du minibus "Rabbit» VW. Faisant siennes les analyses de Jürgen Habermas, Ronda Hauben voit dans les groupes de discussion un espace intermédiaire entre l'état et la société civile, à même de réinsuffler une nouvelle vitalité à l'un comme à l'autre. Cet espoir est celui qui donne au paradigme de l'anarchie une importance cruciale dans l'imaginaire du réseau. En effet, l'utopie techno-anarchiste, en promettant de rénover le lien social sur la base de l'égalité, contribue aux connotations positives du réseau qui nous permettent de nous faire insensiblement à sa présence et d'élaborer des pratiques grâce auxquelles chacun de nous peut se l'approprier, le recréer ou le détourner (Guattari $1991: 2$ ).

\section{Sociabilité}

21 Outre la généralisation du débat démocratique et la mise en valeur de l'égalité, le paradigme de l'anarchie permet de faire de l'Internet la voie d'accès à de nouvelles formes de vie communautaire fondées sur les affinités électives et la liberté. Dans cette optique, l'Internet crée un espace public supplémentaire où peuvent venir s'éprouver les idées nouvelles, se confronter les interprétations du monde. Ces relations sont censées être authentiques précisément parce qu'elles se situent en dehors des conventions sociales et qu'elles sont donc non médiatisées par les usages et les convenances; elles sont censées s'ordonner autour d'une communauté d'intérêts ou de goûts: elles constituent alors une myriade de micro-appartenances telles que le goût pour le rock « $n$ roll ou l'intérêt pour le postmodernismeet à ce titre elles sont censées échapper aux tirages et aux négociations souvent malaisées qui accompagnent les échanges sociaux habituels : des relations sociales épurées, débarrassées de tout déchet et qui en outre permettraient de rompre sans difficulté l'isolement produit par la société contemporaine ${ }^{5}$ 
. L'Internet permettrait ainsi la refondation d'un lien social basé sur une convivialité sans mélange, sur une sociabilité réinventée, et serait ainsi à même d'aboutir à la création d'une société virtuelle plus morale et plus satisfaisante que la société actuelle.

\section{Le paradigme spatio-temporel}

Nombreux sont ceux pour lesquels « le Net nie la géométrie [...], il est fondamentalement et profondément anti-spatial» (Mitchell $1995:$ ch.2.1, 1) ${ }^{6}$. Cette perception repose sur la contraction apparente que l'Internet fait subir à l'espace : lorsqu'on tape une adresse URL ${ }^{7}$, on n'a aucun moyen de savoir avec précision où se situe physiquement l'ordinateur qui héberge le site; d'ailleurs, ce n'est pas la distance qui sera déterminante dans la vitesse de chargement du site, mais bien la puissance de la configuration ordinateur/modem dont on dispose, de celle dont le serveur distant dispose et du niveau d'encombrement de la bande passante. D'où la perception de l'Internet comme un déni de la dimension spatiale.

L'Internet semble modifier également la perception de la durée: les paquets d'information voyagent à une vitesse théorique proche de celle de la lumière et cette promesse d'instantanéité fait que le temps semble se contracter, nous ramenant à un éternel présent tout en détruisant nos catégories habituelles, où le passé est actualisé grâce à la mémoire. La quasi-instantanéité qui caractérise l'obtention d'information serait alors un déni de la dimension temporelle grâce à laquelle une pensée peut se déployer et produire du sens. Par voie de conséquence, l'instantanéité est perçue comme une menace pour le politique, car elle forclôt toute possibilité de réaction réfléchie parce que différée, retirant alors au domaine politique l'une de ses dimensions essentielles, le temps.

À l'inverse, la communication interpersonnelle qui suppose, selon le schéma classique en face-à-face, la simultanéité des échanges, se voit affectée d'un temps d'attente entre le stimulus et la réaction : intrusion de la discontinuité, donc, dans la fluidité de l'échange idéal, et cette discontinuité permet d'échapper à la nécessité d'une réaction immédiate : la parole de l'autre verrait ainsi son impact neutralisé par l'a-synchronie. C'est donc paradoxalement à la fois l'instantané et le différé qui posent problème.

\section{Fonctions des conflits d'imaginaires}

Dès lors que l'on s'intéresse aux discours qui ont accompagné les premières technologies modernes de la communication, on observe de saisissantes convergences avec celui dont nous venons de décrire les grands traits. Comme l'ont démontré François Barbier et Catherine Bertho Lavenir (1996), le discours qui met en relation moyens de communication et culture remonte en France à un texte officiel de 1792, «L'instruction pour les directeurs des Postes ", qui énumère les bienfaits attendus des services postaux. Le télégraphe a également suscité des attentes sociales fortes aussi bien que la méfiance (Carey 1989), selon des modalités qui rappellent celles qui ont actuellement cours pour l'Internet. François Barbier et Catherine Bertho Lavenir précisent ainsi que la crainte de voir des informations illicites circuler plus facilement avait empêché «le ministre de l'Intérieur de Louis-Philippe d'ouvrir l'utilisation du télégraphe Chappe aux particuliers » (1996: 332). Ce n'est qu'en 1894, soit un siècle après l'invention du télégraphe aérien Chappe, que le télégraphe électrique est accessible au public (Breton \& Proulx 1993 : 68). En Grande-Bretagne, le télégraphe engendre un discours sur l'aptitude de cette 
technologie à resserrer les liens entre le centre et la périphérie ainsi qu'une réflexion sur la question du contrôle de l'information (Rhys-Morus 1998 : 228-9). Une partie d'échecs jouée par l'intermédiaire du télégraphe en 1845 , entre deux joueurs situés à cent miles l'un de l'autre, est interprétée comme un signe d'intelligence de la machine elle-même ( ibid. : 227), ce qui nous renvoie au discours contemporain sur l'ordinateur et le réseau.

Le téléphone produit un discours supplémentaire sur la communication et la société. Plusieurs grandes lignes se dégagent, curieusement proches de celles que nous avons identifiées : la crainte d'une transformation dommageable et irrémédiable des modes de communication traditionnels en face-à-face, la crainte de la transparence des communications, donnant lieu à un contrôle social généralisé ; à l'opposé, cependant, le téléphone est perçu comme un instrument propre à favoriser l'instauration de nouvelles communautés, plus harmonieuses car plus ouvertes et plus démocratiques (ibid. : 65). Un juge affirme en 1891 que «le téléphone marque le début d'une ère de voisinage sans proximité $»^{8}$. D'autres enfin croient le téléphone à même de constituer des communautés immédiates ${ }^{9}$, selon les principes énoncés par le déterminisme technologique et que l'on retrouve pratiquement inchangés dans les textes que nous avons étudiés. De façon semblable, la radio est portée par un discours qui souligne à la fois ses capacités intégratrices et démocratiques (Czitrom 1982 : 177). Elle est perçue comme l'instrument à même de pallier l'éclatement de la cellule familiale (Flichy $1991: 220$ ). Les mêmes attentes se font jour vis-à-vis de la télévision, puis de l'ordinateur (Quéré 1983).

Cette brève énumération indique avant tout l'extrême longévité des représentations : il n'y a guère de différence entre les textes que nous venons d'étudier et les espoirs et les craintes exprimés un à deux siècles plus tôt. Qu'il s'agisse de 1792 ou de 1997, les discours sont en fait presque complètement superposables, ce qui suggère que le discours appliqué à l'Internet n'est en rien propre à ce média. De cette constatation découlent deux corollaires : d'une part, la singularité du média n'est pas prise en compte, seule subsiste une notion très générale de modernité ; d'autre part, la singularité de chaque locuteur ou de chaque énoncé n'est guère perceptible. Par contre, les représentations sociales de la communication en réseau sont elles, bien présentes.

Ce discours ne possède qu'un lien très lâche avec le réel ; tout vraisemblable qu'il semble être, c'est un discours arbitraire, fondé sur des opinions préconçues issues de stéréotypes sociaux, et que l'on justifie après coup en invoquant tel ou tel aspect de l'Internet, jamais considéré dans sa spécificité. Le discours sur l'Internet est structuré comme une narration romanesque, avec ses héros (les jeunes scientifiques, la culture, l'égalité), ses antagonistes (la contraction de l'espace-temps, l'indifférenciation des sociétés), son grand méchant (les États-Unis et leur impérialisme culturel), ses victimes (les internautes qui deviennent dépendants du réseau), sa toile de fond sociale (l'individualisme, le délitement des structures familiales et politiques traditionnelles) et son temps du récit (les sociétés qui se transforment à grande vitesse). Les métaphores centrales sont celles de la route et de la navigation, toutes deux inséparables de la notion d'aventure et de quête, ainsi que celle du monde alternatif, de la cité idéale, qui représentent le but de la quête. Lorsqu'on met ces éléments en relation sur le mode narratif, on obtient un récit de type apocalyptique - récit d'une catastrophe annoncée, mais aussi d'un renouveau - qui, pour inquiétant qu'il soit, a pour ceux qui l'emploient le mérite de créer un début, un milieu et une fin, donc un sens et du sens, parmi tous ces éléments disjoints. Ce qui est à l'œuvre est donc un processus de familiarisation (Moscovici 1998 : 13). En d'autres termes, il s'agit de rattacher le phénomène nouveau à un phénomène connu, et ainsi de trouver 
les outils conceptuels nécessaires à son appréhension puis à son appropriation. C'est une façon de structurer la réalité, de lui donner un sens même lorsqu'il s'agit d'événements qui semblent traversés par l'altérité. Ils parviennent à constituer l'Internet en objet de discours doté de caractéristiques propres, en un mot, à lui donner une existence.

Or, ce discours vraisemblable et plausible demeure profondément insatisfaisant et présente, notamment, un déficit d'adéquation au réel. La théorie de l'information telle que Norbert Wiener l'expose offre un début d'explication à ce phénomène. Cette théorie pose en effet que le cliché ou le lieu commun sont peu éclairants parce qu'ils sont singulièrement soumis à l'entropie. En d'autres termes, «plus le message est probable, moins il fournit d'information" (Wiener $1962: 24$ ) (italiques dans le texte). Nous pouvons donc en déduire que le rôle des représentations stéréotypées est d'apprivoiser l'étrange, de forcer la réalité à se conformer à un schéma reconnaissable et qu'à ce titre il la masque bien plus qu'il ne la révèle. Nous pouvons même aller jusqu'à dire que la fonction de masquage l'emporte sur la fonction explicative du moins au début de la diffusion de masse de l'Internet.

Ce processus de familiarisation obscurcit la perception de la disparité et, en même temps, permet de créer une continuité symbolique entre innovations passées et technologies de pointe. En d'autres termes, la représentation stéréotypée joue le rôle d'intertexte: de même que chaque œuvre littéraire nouvellement créée a pour intertextualité la totalité des œuvres qui l'ont précédée, de même les technologies qui furent nouvelles et le discours stéréotypé sur elles constituent l'intertexte dans lequel l'Internet vient s'inscrire. Cet univers discursif sert de médiateur au réseau, lui permet d'être visible, sinon compris. Et dans ce discours pré-construit où s'insère l'Internet, la coexistence des discours apocalyptiques et des discours optimistes perdure en dépit de tous les démentis que peut leur infliger la réalité des pratiques sociales. Cette coexistence nous amène à penser que les deux versants, positifs et négatifs, doivent se concevoir en synergie plutôt qu'en opposition: du fait même de leur simultanéité, ils se renforcent l'un l'autre, ils offrent un espace discursif à la critique sociale des NTIC tout en assurant simultanément leur acculturation et leur diffusion.

31 Les conflits d'imaginaires évoqués auparavant produisent ainsi des discours dont l'importance est cruciale pour la diffusion des innovations et singulièrement pour la diffusion des nouvelles technologies de la communication. En d'autres termes, l'imaginaire et le symbolique ont sur la réalité des répercussions certaines. Si les énoncés que nous avons étudiés reproduisent avec tant de clarté deux interprétations conflictuelles majeures, c'est que la lutte pour le sens bat son plein dans la société française contemporaine, de même que dans les sociétés britannique et américaine. Le développement de l'Internet, par sa rapidité, semble remettre en cause non seulement des positions bien établies, comme celles des médias traditionnels, mais encore, et bien plus profondément, modifier la vision que ces sociétés ont d'elles-mêmes. L'Internet sert alors de lieu nodal des contraires, focalisant sur lui les luttes pour le sens de la société tout entière. Ces luttes ne cesseront pas de sitôt. 


\section{BIBLIOGRAPHIE}

Barbier, Frédéric et Catherine Bertho Lavenir. 1996. Histoire des médias : de Diderot à Internet. Paris : A. Colin/Masson.

Bimber, Bruce. 1998. « The Internet and political transformation: Populism, community and accelerated pluralism ». Polity XXXI/1, Fall, 133-160.

Bourdieu, Pierre. 1984. Questions de sociologie. Paris : Minuit.

Breton Philippe et Serge Proulx. 1993. L'explosion de la communication. Paris : La Découverte.

Carey, James W. 1989. Communication as Culture: Essays on Media and Society. Boston : Unwin Hyman.

Czitrom, Daniel J. 1982. Media and the American Mind: From Morse to McLuhan. Chapel Hill : University of North Carolina Press.

Flichy, Patrice. 1991. Une histoire de la communication moderne : espace public et vie privée. Paris : La Découverte.

Grossman, Lawrence K. 1995. The Electronic Republic: Reshaping Democracy in the Electronic Age. NewYork : Viking Press.

Guattari, Félix. 1991. « Pour une éthique des médias ». Le Monde, 6 novembre, 2.

Hauben, Michael \& Ronda Hauben. 1997. Netizens: On the History and Impact of Usenet and the Interne t. Piscataway, NJ : Computer Society Publications.

Jeanneret, Yves. 1998. «Ce que l'écran change à l'écrit ». Sciences humaines, hors série 21, juinjuillet, 37.

Marvin, Carolyn. 1990. When Old Technologies Were New. New York : Oxford University Press.

Mathias, Paul.1997. La cité Internet. Paris : Presses de Sciences-Po.

Mitchell, William J. 1995. City of Bits. Cambridge, MA : The MIT Press.

Moscovici, Serge. 1998. «Comment voit-on le monde ? Représentations sociales et réalités ». Sciences humaines, hors série 21, juin-juillet, 13.

Nguyen, Dan T. \& Jon Alexander. 1996. «The coming of cyberspacetime and the end of polity ». In Shields Rob (dir.), Cultures of Internet: Virtual Spaces, Real Histories, Living Bodies. Londres : Sage.

Quéré, Louis. 1983. «Qu'est-ce qu'une révolution de la communication ». Action et recherches sociales : langages et médiations 10/1, 49-62.

Rhys-Morus, Iwan. 1998. Frankenstein's Children : Electricity, Exhibition and Experiment in Early Nineteenth Century London. Princeton, N.J. : Princeton University Press.

Serfaty, Viviane. 1999. «L'Internet : fragments d'un discours utopique ». Communications et Langages 119, janvier-mars, 106-117.

Shade, Leslie R. 1996. "Is there free speech on the Net ? Censorship in the global information infrastructure ». In Shields Rob (dir.), Cultures of Internet: Virtual Spaces, Real Histories, Living Bodies. Londres : Sage. 
Wiener, Norbert. 1962. Cybernétique et société. Paris : Éditions des Deux-Rives.

\section{NOTES}

1. Communication présentée au $\mathrm{XL}^{\mathrm{e}}$ colloque de la SAES, Angers, 19-21 mai 2000. Atelier Recherche et Nouvelles Technologies.

2. Pierre Bourdieu a écrit «Quelques propriétés des champs » : [...] ce n'est pas par hasard qu'un des indices les plus sûrs de la constitution d'un champ est [...] l'apparition d'un corps de conservateurs des vies - les biographes - et des oeuvres - les philologues, les historiens de l'art et de la littérature, qui commencent à archiver les esquisses [...] , autant de gens qui ont partie liée avec la conservation de ce qui se produit dans le champ, qui ont intérêt à conserver et à se conserver conservant » $(1984: 16)$. Merci à Jean-Baptiste Legavre, qui a attiré mon attention sur ce texte.

3. Voir Viviane Serfaty (1999) pour une étude approfondie de ces notions.

4. Jon Postel, RFC 1591, March 1994 https://tools.ietf.org/html/rfc1591.

5. "Contre la solitude, la connexion avec des millions d'internautes", publicité parue dans Le Nouvel Observateur, 1769, 1-7 octobre 1998, 94-99.

6. "The Net negates geometry, [...] it is fundamentally and profoundly antispatial », Extraits disponibles <http://mitpress.mit.edu/e-books/City_of_Bits/Electronic_Agoras/ SpatialAntispatial.html>.

7. URL : Uniform Resource Locator, traduit par « adresse universelle».

8. «...[the] epoch of neighbourship without propinquity ", Anon., " Epoch-Making Inventions of America », Electrical Review, April 18, 1891, 109 cité par Carolyn Marvin (1990: 66, notre traduction).

9. «technical devices would be able to create communities on the spot» (Marvin 1990: 66, notre traduction).

\section{RÉSUMÉS}

L'Internet suscite des interprétations contradictoires mais il est aussi l'objet d'une immense quantité de discours qui véhiculent un ensemble de représentations sociales. Leur étude permet d'identifier les structures profondes de l'imaginaire qui sous-tend l'Internet. L'utopie et l'antiutopie sont les deux notions antinomiques au travers desquelles l'Internet est perçu et qui sont à l'origine de véritables conflits d'imaginaires. L'étude des discours sur l'Internet fait également apparaître trois grands paradigmes traversés par l'ambivalence puisqu'ils se rattachent au clivage utopie/anti-utopie. Il s'agit du paradigme culturel et communicationnel, du paradigme de l'anarchie et du paradigme spatio-temporel. L'exploration de l'histoire des idées permet de retrouver les origines de cette ambivalence et d'identifier l'existence de représentations sociales stéréotypées présentes sous des formes quasiment identiques depuis l'invention des premiers outils de communication modernes au XVIII ${ }^{\mathrm{e}}$ siècle. Le sens et la fonction de ces conflits d'imaginaire ainsi que des stéréotypes sont analysés dans leur rapport aux NTIC et singulièrement à l'Internet. 
The Internet gives rise to a variety of contradictory interpretations, but it is also the subject of a vast amount of discourse conveying a variety of social representations. By studying these representations, we can identify the deep-seated structures of the common imagination on which the Internet is based. Utopia and anti-Utopia are the two antitomic notions through which the Internet is perceived and which lie at the heart of some very real conflicts of the imagination. The study of discourse on the Internet also highlights three major paradigms, ambivalent to a certain extent since they are linked with the Utopia / anti-Utopia divide. These are the cultural and communicational paradigm, the paradigm of anarchy and the space-time paradigm. The origins of this ambivalence can be traced back to the history of ideas as we can identify the existence of virtually the same stereotypical social representations since the invention of the first tools of modern communication in the 18th century. We analyse the meaning and the function of these conflicts of the imagination as well as stereotypes in relation to NICT and in particular to the Internet.

\section{INDEX}

Mots-clés : communication, imaginaire, Internet, NTIC, stéréotype, utopie

Keywords : communication, imaginary, Internet, NICT, stereotype, utopia

\section{AUTEUR}

VIVIANE SERFATY 